\title{
Catalytic upgrading of coal pyrolysis gaseous tar over hierarchical Y-type zeolites synthesized using microwave hydrothermal method
}

Peng Lv, Lunjing Yan, Yan Liu, Meijun Wang, Weiren Bao*, Fan Li*

State Key Laboratory Breeding Base of Coal Science and Technology Co-founded by

Shanxi Province and the Ministry of Science and Technology, Taiyuan University of

Technology, Taiyuan 030024, China

\section{Supporting Information}

Figure S1. $\mathrm{NH}_{3}$-TPD curve of Y-type zeolites prepared using different amounts of template.

Figure S2. CP/MAS ${ }^{13} \mathrm{C}$ NMR fitted spectrum of the coal sample.

Table S1. Proximate and ultimate analyses of the coal samples.

Table S2. Acidity distribution of the samples obtained using $\mathrm{NH}_{3}-\mathrm{TPD}$.

Table S3. Chemical shifts of different types of carbon structures. 


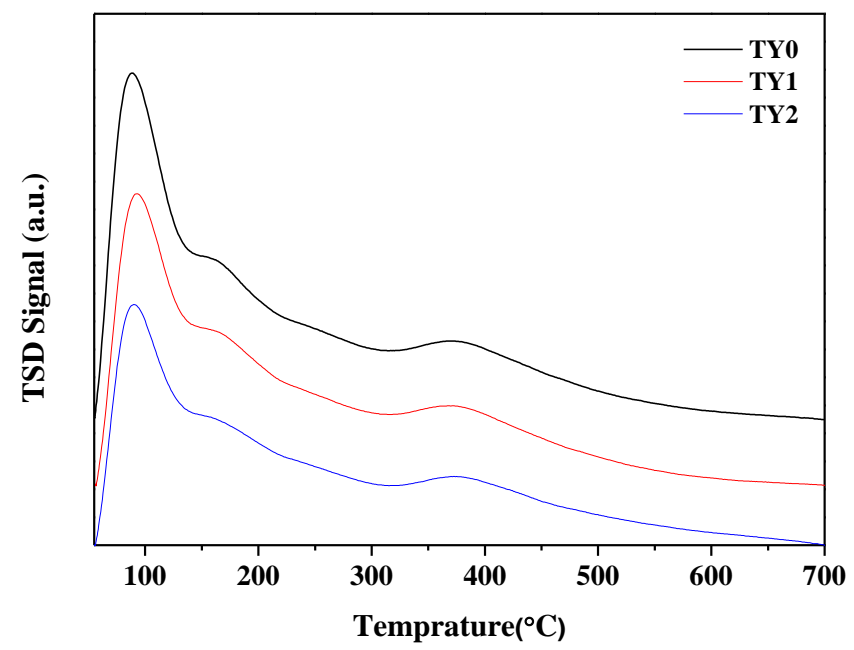

Figure S1. $\mathrm{NH}_{3}$-TPD curve of Y-type zeolites prepared using different amounts of template. 


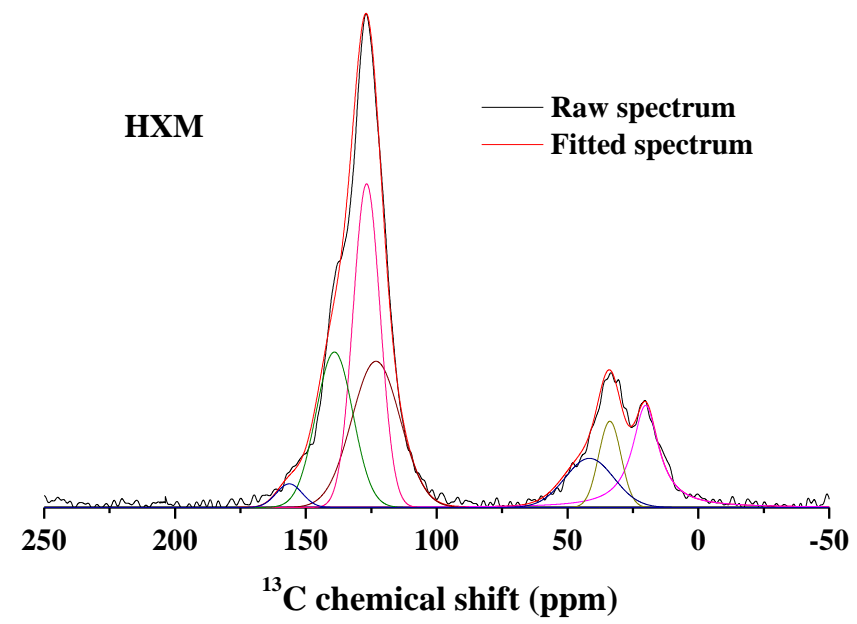

Figure S2. $\mathrm{CP} / \mathrm{MAS}{ }^{13} \mathrm{C} \mathrm{NMR}$ fitted spectrum of the coal sample. 
Table S1. Proximate and ultimate analyses of the coal samples.

\begin{tabular}{|c|c|c|c|c|c|c|c|c|}
\hline \multirow{2}{*}{ Samples } & \multicolumn{3}{|c|}{ Proximate analysis wt. $/ \%$} & \multicolumn{5}{|c|}{ Ultimate analysis wt. $/ \%$, daf } \\
\hline & $\mathrm{M}_{\mathrm{ad}}$ & $A_{d}$ & $\mathrm{~V}_{\mathrm{daf}}$ & $\mathrm{C}$ & $\mathrm{H}$ & $\mathrm{O}^{*}$ & $\mathrm{~N}$ & $\mathrm{~S}$ \\
\hline PSM & 2.2 & 18.3 & 37.2 & 80.4 & 5.2 & 11.9 & 1.4 & 1.1 \\
\hline FCM & 2.3 & 7.8 & 40.4 & 82.1 & 5.2 & 8.1 & 1.4 & 3.2 \\
\hline HXM & 0.5 & 9.0 & 22.9 & 86.1 & 4.7 & 7.1 & 1.7 & 0.4 \\
\hline
\end{tabular}

Note: ad, air-dried basis; d, dry basis; daf, dry and ash-free basis; *by difference 
Table S2. Acidity distribution of the samples obtained using $\mathrm{NH}_{3}-\mathrm{TPD}$.

\begin{tabular}{lccc}
\hline & \multicolumn{3}{c}{ Amount $(\mathrm{mmol} / \mathrm{g})$} \\
\cline { 2 - 4 } Samples & Weak acid & Medium and strong acid & Total acid \\
\hline TY0 & 0.90 & 1.58 & 2.48 \\
TY1 & 0.86 & 1.51 & 2.37 \\
TY2 & 0.71 & 1.35 & 2.07 \\
\hline
\end{tabular}


Table S3. Chemical shifts of different types of carbon structures.

\begin{tabular}{|c|c|c|c|}
\hline Chemical shift (ppm) & Functional groups & Carbon functionality & Symbols \\
\hline $0-25$ & $-\mathrm{CH}_{3}$ & Methyl & $\mathrm{f}_{\mathrm{al}}^{1}$ \\
\hline $25-50$ & & Methylene & $\mathrm{f}_{\mathrm{al}}^{2}$ \\
\hline $50-67$ & $\mathrm{CH}_{3} \mathrm{O}-$ & Methoxy & $\mathrm{f}_{\mathrm{al}}^{\mathrm{ol}}$ \\
\hline $67-90$ & $\mathrm{OH}, \mathrm{CH}_{2} \mathrm{OH}$ & $\begin{array}{l}\text { Oxy-methine ,Saccharide, } \\
\text { Alcohol, Ether }\end{array}$ & $\mathrm{f}_{\mathrm{al}}^{\mathrm{o} 2}$ \\
\hline $90-129$ & & $\begin{array}{c}\text { Aromatic atoms bound to } \\
\text { hydrogen }\end{array}$ & $\mathrm{f}_{\mathrm{a}}^{1}$ \\
\hline $129-137$ & & $\begin{array}{l}\text { Bridging ring junction } \\
\text { aromatic carbon }\end{array}$ & $\mathrm{f}_{\mathrm{a}}^{2}$ \\
\hline $137-148$ & & Branched aromatic carbon & $\mathrm{f}_{\mathrm{a}}^{3}$ \\
\hline $148-171$ & & Oxy-aromatic carbon & $\mathrm{f}_{\mathrm{a}}^{\mathrm{ol}}$ \\
\hline $171-187$ & $-\mathrm{COOR} / \mathrm{H}$ & Carboxyl, Ester, Quinone & $\mathrm{f}^{\text {co }}$ \\
\hline $187-220$ & $\mathrm{C}_{\mathrm{C}}^{\mathrm{C}}=\mathrm{O}$ & Ketone, Quinine, Aldehyde & $\mathrm{f}^{\mathrm{co}}$ \\
\hline
\end{tabular}

